\title{
Terminologia especializada de enfermagem no cuidado às pessoas vivendo com aids
}

Specialized nursing terminology in care of people infected with AIDS

Terminología especializada de enfermería en el cuidado de personas que viven con sida

Bárbara Coeli Oliveira da Silva ${ }^{1}$ ic https://orcid.org/0000-0002-2933-0930

Rafael Medeiros Santos ${ }^{1}$ ii https://orcid.org/0000-0002-9504-8191

Fernanda Rafaela dos Santos ${ }^{1}$ id https://orcid.org/0000-0002-6538-9411

Thais Monteiro da Silva Padiliha ${ }^{2}$ io https://orcid.org/0000-0003-0165-3102

Olga Alice Alencar Moreira ${ }^{1}$ io https://orcid.org/0000-0002-5625-7011

Elieze da Silva Tavares ${ }^{1}$ io hitps://orcid.org/10000-0002-7444-7898

Richardson Augusto Rosendo da Silva ${ }^{1}$ io hittps://orcid. org/0000-0001-6290-9365

\section{Como citar:}

Silva BC, Santos RM, Santos FR, Padilha TM,

Moreira AO, Tavares ES, et al. Terminologia

especializada de enfermagem no cuidado às

pessoas vivendo com AIDS. Acta Paul Enferm. 2021;34:eAPE03122.

DOI

http://dx.doi.org/10.37689/actaape/2021A003122

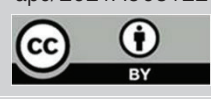

Descritores

Cuidados de enfermagem; Terminologia padronizada em enfermagem; Síndrome de imunodeficiência adquirida; HIV

Keywords

Nursing care; Standardized nursing terminology; Acquired immunodeficiency syndrome; HIV

Descriptores

Atención de enfermería; Terminología normalizada de enfermería; Síndrome de imunodeficiência

adquirida; $\mathrm{VIH}$

\section{Submetido \\ 26 de Novembro de 2019 \\ Aceito \\ 3 de Junho de 2020}

Autor correspondente

Bárbara Coeli Oliveira da Silva E-mail: barbaracoeli@outlook.com

\section{Resumo}

Objetivo: Construir e validar quanto ao conteúdo uma terminologia especializada de Enfermagem, no cuidado a adultos vivendo com aids, fundamentado no Modelo de Sete Eixos da Classificação Internacional para a Prática de Enfermagem.

Métodos: Estudo metodológico, realizado em um Hospital Escola no Nordeste do Brasil. Seguiram-se as seguintes etapas: extração dos termos de prontuários de pessoas vivendo com aids; normalização; mapeamento cruzado entre os extraídos e os constantes na Classificação Internacional para a Prática de Enfermagem; distribuição destes nos sete eixos e validação de conteúdo por meio de índice de concordância entre enfermeiros peritos.

Resultados: Extraíram-se 2.000 termos. A normalização resultou em 557 termos pertinentes, estando 319 constantes e 238 não constantes na Classificação Internacional para a Prática de Enfermagem. Foram validados pelos peritos 522 termos, sendo 319 constantes e 203 não constantes, os quais atingiram um índice de concordância $\geq 0,80$.

Conclusão: 0 estudo permitiu identificar e validar os termos utilizados por enfermeiros na assistência às pessoas vivendo com aids, o qual subsidiará as etapas subsequentes à construção de um subconjunto terminológico para informação e comunicação à prática de Enfermagem.

\section{Abstract}

Objective: To build and validate, in terms of content, a specialized nursing terminology in care of adults infected with AIDS, based on the Seven-Axis Model of the International Classification for Nursing Practice.

Methods: A methodological study carried out at a teaching hospital in northeastern Brazil. The following steps followed extraction of terms from the medical records of people infected with AIDS; normalization; crossmapping between extracted and constant in the International Classification for Nursing Practice; distribution in seven axes and content validation through a concordance index among expert nurses.

Results: Two thousand terms have been extracted. Normalization resulted in 557 pertinent terms, 319 of which were constant and 238 not included in the International Classification for Nursing Practice. Five hundred and twenty-two terms were validated by experts, of which 319 were constant and 203 were not constant, which reached a concordance index $\geq 0.80$.

Conclusion: This study allowed to identify and validate the terms used by nurses in assisting people infected with AIDS, which will subsidize the steps subsequent to the construction of a terminological subset for information and communication to nursing practice. 


\section{Resumen}

Objetivo: Elaborar y validar, en cuanto al contenido, una terminología especializada de enfermería en el cuidado de adultos que viven con sida, fundamentada en el Modelo de Siete Ejes de la Clasificación Internacional de la Práctica de Enfermería.

Métodos: Estudio metodológico, realizado en un hospital universitario en la región nordeste de Brasil. Se llevaron a cabo las siguientes etapas: extracción de los términos de historias clínicas de personas que viven con sida; normalización; mapeo cruzado entre los términos extraídos y los que constan en la Clasificación Internacional de la Práctica de Enfermería; distribución de estos en los siete ejes y validación de contenido mediante el índice de concordancia por enfermeros peritos.

Resultados: Se extrajeron 2.000 términos. La normalización dio como resultado 557 términos pertinentes, de los cuales 319 constaban en la Clasificación Internacional de la Práctica de Enfermería y 238 no. Los peritos validaron 522 términos, de los cuales 319 constaban y 203 no constaban, que alcanzaron un índice de concordancia $\geq 0,80$.

Conclusión: El estudio permitió identificar y validar los términos utilizados por enfermeros en la atención a personas que viven con sida y contribuirá con las etapas subsiguientes de la construcción de un subconjunto terminológico para información y comunicación en la práctica de enfermería.

\section{Introdução}

A aids caracteriza-se como um importante problema de saúde pública mundial. ${ }^{(1)}$ Segundo o relatório publicado no ano de 2019 pelo Programa Conjunto das Naçóes Unidas sobre HIV/aids (UNAIDS), tem-se que em 2018 existia 37,9 milhões de pessoas vivendo com essa enfermidade no mundo, sendo 966.058 mil no Brasil. ${ }^{(2)}$

Por ser considerada uma doença de caráter crônico, requer dos envolvidos diretamente com o cuidado açóes que influenciem positivamente na vida das pessoas vivendo com aids. ${ }^{(3)}$ Nesse sentido, o enfermeiro possui um importante papel no cuidado dessas pessoas, desenvolvendo açóes de promoção, proteção e reabilitação da saúde, com ênfase na adesão ao tratamento e atuação das pessoas vivendo com aids no autocuidado. ${ }^{(4)}$

Para tanto, cabe ao enfermeiro antes e durante o cuidado a essa população desenvolver habilidades técnico-científicas que favoreçam a organização e Sistematização da Assistência de Enfermagem (SAE). ${ }^{(3)}$ De acordo com a Resolução COFEN-358/2009, a SAE "organiza o trabalho profissional quanto ao método, pessoal e instrumentos, tornando possível a operacionalização do Processo de Enfermagem (PE)", sendo este conceituado como 'um instrumento metodológico que orienta tanto o cuidado profissional de Enfermagem quanto a documentação da prática profissional". ${ }^{(5)}$

Além disso, evidencia-se na literatura a importância da utilização dos sistemas de classificação durante a aplicação em todas as fases do PE por meio das terminologias existentes no campo da enfermagem que servem para unificar a linguagem da profissão favorecendo o aumento, a melhoria da documentação e qualidade dos registros de enfermagem. ${ }^{(6)}$
Entre os sistemas de classificação em Enfermagem, ressalta-se a Classificação Internacional para a Prática de Enfermagem (CIPE $)$. Sua estrutura de termos e definições permite a coleta, a descrição e a documentação sistemática das etapas do $\mathrm{PE}$ por meio da menção, relação e encadeamento dos episódios que acontecem na prática profissional. ${ }^{(7)}$

Como não existem classificações específicas para todas as áreas de atuação do enfermeiro, é necessário coletar e codificar termos utilizados pela Enfermagem em clientes e áreas específicas, que poderão ser utilizados posteriormente para a estruturação de subconjuntos terminológicos $(\mathrm{ST}){ }^{(8)}$ Estes são definidos como um conjunto de enunciados de diagnósticos, resultados e intervençôes de enfermagem para uma determinada área selecionada ou de especialidade do cuidar em enfermagem com base no Modelo de Sete Eixos da CIPE ${ }^{\circledast}{ }^{(9)}$ Os ST são essenciais para a prestação de cuidados individualizados aos clientes e às suas famílias, como uma referência acessível para os enfermeiros. $^{(10)}$

Ademais, esses subconjuntos auxiliam na geração dos indicadores diagnósticos, sendo úteis na prática, fazendo com que os enfermeiros possam quantificar e qualificar os resultados por meio das suas intervençóes, buscando por meio destes uma melhoria na qualidade do seu cuidado prestado. ${ }^{(10)}$

Diante do panorama apresentado, buscou-se por produçôes científicas sobre uma terminologia especializada de Enfermagem para pessoas vivendo com aids, nas bases de dados: Literatura LatinoAmericana e do Caribe em Ciências da Saúde, Medical Literature Analysisand Retrieval System Online, Base de Dados de Enfermagem, Scopus, Cumulative Index to Nursingand Allied Health 
Literature, Scientific Electronic Library Online e Web of Science.

Identificou-se escassez de estudos relacionados à temática, encontrando-se apenas uma pesquisa com enfoque em mulheres idosas com HIV/ aids ${ }^{(11)}$ que partiu de um publicação do Ministério da Saúde, voltada para o sexo feminino, não sendo ideal, portanto, a sua utilizaçáo no presente estudo, pois buscou-se por uma Terminologia especializada de enfermagem que abrangesse também a população adulta jovem acometida por aids e que fosse extraída de registros de enfermagem. As demais pesquisas tratavam sobre termos específicos relacionados à clínica médica, cirúrgica, unidade de terapia intensiva neonatal e de adultos, reabilitação físico-motora, ostomizados, pessoas com lesão por pressão, hanseníase e cuidados paliativos. (12-20) Considerando-se que mulheres idosas possuem peculiaridades inerentes ao seu ciclo vital e gênero, o que as diferenciam da população adulta jovem de ambos os sexos, justifica-se a construção de uma terminologia especializada para pessoas adultas com aids.

É importante ressaltar que os registros de Enfermagem evidenciam o cuidado prestado, no entanto, quando são apresentados de forma incompleta ou imprecisa, podem ser interpretados erroneamente. ${ }^{(12)}$ Já o uso de uma terminologia especializada permite detectar conceitos que auxiliam na construção de diagnósticos, resultados e intervençôes de Enfermagem, colaborando, desse modo, com o cuidado prestado pelo Enfermeiro. ${ }^{(20)}$

A partir desse contexto, questiona-se: Que termos são utilizados pelos enfermeiros no cuidado às pessoas vivendo com aids? Quais podem constituir uma terminologia especializada que oriente a prática clínica e o registro efetivo de dados de enfermagem na atenção a essas pessoas, após seu processo de extração, normalização e validaçáo?

Diante do exposto, este estudo objetivou construir e validar quanto ao conteúdo uma terminologia especializada de Enfermagem, no cuidado a adultos vivendo com aids, fundamentado no Modelo de Sete Eixos da Classificação Internacional para a Prática de Enfermagem.

\section{Métodos}

Trata-se de um estudo metodológico, desenvolvido com os registros de enfermeiros dos prontuários de pessoas vivendo com aids. A pesquisa foi realizada em um hospital público no Nordeste do Brasil, referência no tratamento de doenças infectocontagiosas.

Foram selecionados os registros feitos pelos enfermeiros dos prontuários físicos de pessoas vivendo com aids, entre janeiro e dezembro de 2015, do referido hospital, disponibilizados pelo Serviço de Arquivo Médico e Estatístico do hospital. Neles, há um espaço específico para os registros de Enfermagem. No período estabelecido, foram internados no referido hospital 198 pacientes com aids, conforme dados obtidos pelo Serviço de Arquivos Médicos do hospital.

Para determinar o número de prontuários necessários para o estudo, utilizou-se o cálculo de amostra para populaçôes finitas com erro amostral de 10\%, nível de confiança de $95 \%\left(Z_{\infty}=1,96\right)$ e prevalência de $50 \%$, constituindo-se, assim, uma amostra de 65 prontuários. ${ }^{(21)}$ Ressalta-se que, por não ter sido encontrado um estudo que estimasse a prevalência geral de terminologia especializada em enfermagem para pacientes adultos com aids internados em hospital, considerou-se um valor conservador de $50 \%$.

De modo a evitar o viés de seleção, utilizou-se o processo de amostragem sistematizada, sendo selecionado um prontuário a cada três listados sequencialmente. $\mathrm{O}$ intervalo a cada três foi determinado pela divisão do total de pacientes internados no ano de 2015 (198) pela amostra de prontuários (65) necessários para o estudo.

Adotaram-se como critério de inclusão prontuários com registro dos enfermeiros durante período de internação de pessoas com idade maior ou igual a 18 anos, vivendo com aids nas enfermarias, e como critério de exclusão os prontuários com ausência de registro em pelo menos um turno da internação, caracterizando descontinuidade dos registros de Enfermagem.

A coleta de dados nos prontuários ocorreu no período de janeiro a março de 2016 e foi realizada pela pesquisadora principal, alunos e colaboradores pertencentes ao grupo de pesquisa de uma instituição fede- 
ral de ensino, sendo supervisionada pelo orientador do estudo. Fez-se um treinamento prévio para seleção dos colaboradores e padronizaçáo da coleta de dados. Assim, realizou-se um minicurso de 20 horas, por meio da metodologia da problematização, com discussões de textos, resolução de estudos de casos clínicos e simulação de extração de termos de registros de enfermagem. Os temas abordados foram: a CIPE', normalização e mapeamento cruzado de termos, elaboração de terminologias especializadas de Enfermagem e assistência de enfermagem às pessoas vivendo com aids.

Para o cálculo do número de peritos, adotou-se o referencial metodológico proposto pelos autores, ${ }^{(22)}$ utilizando-se a fórmula: $\mathrm{n}=\mathrm{Z} \alpha 2{ }^{*} \mathrm{P}^{*}(1-\mathrm{P}) /$ $\mathrm{e}^{2}$, em que " $Z \alpha$ " se refere ao nível de confiança adotado (95\%); "P" representa a proporção de especialistas que indicam a adequação dos itens (85\%); e "e" representa a diferença proporcional aceitável em relação ao que pode ser esperado (15\%). Portanto, como o coeficiente $Z \alpha$ de acordo com o padrão normal de distribuição assume um valor tabulado de 1.96 para um nível de confiança de 95\%, o cálculo do tamanho amostral ao final foi definido por $\mathrm{n}=1.962^{*} 0.85^{*} 0.15 / 0.152=22$ especialistas.

Para a identificação dos peritos, utilizaram-se os critérios de um estudo, ${ }^{(1)}$ de forma adaptada. Assim, participaram da pesquisa enfermeiros denominados peritos, de um grupo de pesquisa sobre estudos da CIPE $^{\circ}$ de uma Universidade Federal no Brasil, necessitando apresentar pelo menos dois dos seguintes critérios: enfermeiro com mestrado ou doutorado; pesquisadores da área da CIPE; enfermeiros assistenciais da área de HIV/aids; e possuir publicação de artigo sobre terminologia ou processo de Enfermagem utilizando a CIPE:

Após o refinamento dos critérios estabelecidos, bem como a possibilidade de contato com estes, foram convidados para participarem da pesquisa por meio de uma carta convite, via e-mail, enfermeiros de um grupo de pesquisa sobre estudos da CIPE de uma Universidade Federal no Brasil. Diante do aceite para participar, encaminhou-se o Termo de Consentimento Livre e Esclarecido e um formulário estruturado, no formato eletrônico, via Google Forms, contendo os termos. A avaliação do formulário ocorreu em apenas uma rodada.
Dos 22 peritos convidados para participarem do estudo, apenas oito aceitaram, os quais responderam ao instrumento, constituindo-se como peritos do referido estudo. Os peritos eram em sua maioria do sexo feminino (90\%), entre 25 e 35 anos de idade $(78 \%)$, possuíam mestrado em Enfermagem (55\%) e atuavam na área hospitalar $(80 \%)$. O processo de validação ocorreu entre setembro e outubro de 2019.

Ressalta-se que à época, quando o manuscrito foi escrito, estava disponível a versão 2017 da CIPE;, com a qual foi realizada uma primeira validação de conteúdo, sendo, posteriormente, lançada a versão 2019. Considerando que a Norma ISO $12300^{(23)}$ destaca entre seus princípios que os mapeamentos devem ter processos de melhoria contínua, assim como ser mantidos e regularmente atualizados durante seu ciclo de vida, julgou-se necessária a atualização com essa nova versão e, portanto, a realização de um novo mapeamento, que evidenciou divergências entre os termos constantes entre as duas referidas versóes da $\mathrm{CIPE}^{\circ}$ que compóem a terminologia especializada. Nesse sentido, realizou-se uma nova validação de conteúdo em 2019, justificando o intervalo de tempo para conclusão do estudo.

Foram seguidas as etapas recomendadas pelas autoras $^{(24)}$ adaptadas para elaboração de terminologia especializada: 1) processo de extração de termos de prontuários e eliminação das repetiçôes; 2) processo de normalização de termos; 3) processo de mapeamento cruzado entre termos extraídos e os termos constantes na $\mathrm{CIPE}^{\circ}$ versão 2019 ; 4) procedimento de refinamento de termos. As seguintes adaptaçóes foram realizadas: na segunda etapa, incluíram-se adequação de sigla e classificação farmacológica dos medicamentos; na terceira etapa, utilizou-se a CIPE ${ }^{\circ}$ versão 2019.

Para extração dos termos, elaborou-se uma planilha do Microsoft Office Excel'2016 em que constavam duas colunas individualizadas para cada prontuário: a primeira com a transcrição dos parágrafos dos registros e a segunda com os termos extraídos desse parágrafo.

Após a exclusão das repetições realizadas depois da finalização da planilha no Microsoft Office Excel $^{\circ} 2016$ organizada em ordem alfabética, dei- 
xando-se apenas a primeira vez que o termo apareceu e contabilizando o número de repetiçóes, fez-se a normalização dos termos encontrados. Para tanto, realizaram-se a correção ortográfica; uniformização dos tempos verbais, gêneros gramaticais e número; adequação de siglas; e exclusão de expressôes pseudoterminológicas.

Os medicamentos foram agrupados pela sua classe farmacológica, quais sejam: Antibióticos, Antimicóticos, Antivirais, Antiparasitários, Sulfonamidas, Anti-histamínicos, Expectorantes, Broncodilatadores, Cardiotônicos, Betabloqueadores, Vasoconstritores, Vasodilatadores, Anti-hipertensivo, Coagulante, Anticoagulantes, Antiagregante plaquetário, Antilipêmicos, Depressores do sistema nervoso central, Sedativos, Analgésicos opioides, Analgésicos não opioides, Anticonvulsivantes, Antiparkinsoniano, Tranquilizantes, Antidepressivos, Ansiolíticos, Anti-inflamatórios não esteroidais, Antissecretores gástricos, Antiácidos, Antieméticos, Laxantes, Antidiarreicos, Hipoglicemiantes e Diuréticos.

Em seguida foi realizado o mapeamento cruzado com a finalidade de identificar os termos constantes e não constantes na CIPE ${ }^{\circ}$ versão 2019. Nessa fase, utilizou-se a ISO 12300/2006. Assim, realizou-se o cruzamento dos termos extraídos dos prontuários com os da CIPE, a partir do Microsoft Office Access 2016 .

Destaca-se que foram construídas definições operacionais para todos os termos normalizados, utilizando a CIPE;, artigos científicos e dicionários de língua portuguesa e de termos técnicos de saúde, no intuito de facilitar a validação por especialistas. A construção das definiçóes operacionais ocorreu de acordo com as etapas recomendadas pela literatura:(25) 1) desenvolvimento de uma definição preliminar; 2) revisão da literatura; 3) desenvolvimento ou identificaçáo de características específicas; 4) mapeamento do significado do conceito; e 5) afirmação da definição operacional.

Em seguida, organizou-se um formulário contendo os termos, suas alocaçóes nos sete eixos da CIPE $^{\circ}$ e definiçóes, submetido ao processo de validação de conteúdo, alinhado ao Referencial Teórico das necessidades humanas básicas, ${ }^{(26)}$ nos três níveis de vida psíquica: psicobiológico, psicossocial e psicoespiritual, de forma a fundamentar o estudo. Assim, o grupo de enfermeiros peritos pode assinalar se considerava aplicável ou não, bem como inserir sugestôes. Os ajustes/adequaçóes realizadas foram rediscutidos com os peritos.

Por fim, calculou-se o Índice de Concordância (IC) entre os peritos para cada termo por meio da fórmula: $\mathrm{IC}=\mathrm{NC} /(\mathrm{NC}+\mathrm{ND})$, em que $\mathrm{NC}=$ número de concordância e ND = número de discordância. ${ }^{(27)}$ Foram validados os termos que atingiram um IC $\geq 0,80$ entre os peritos.

A pesquisa foi aprovada pelo Comitê de Ética em Pesquisa sob o parecer $n^{\circ} 1.177 .410$ e foram observadas as diretrizes e normas regulamentadoras das pesquisas envolvendo seres humanos.

\section{Resultados}

Foram extraídos 2.000 termos dos registros dos enfermeiros dos prontuários de pessoas vivendo com aids que após o processo de normalização resultaram em 557 termos úteis. Estes foram submetidos ao mapeamento cruzado, totalizando 319 termos constantes e 238 não constantes na CIPE'. Foram validados pelos peritos 522 termos, sendo 319 constantes (Quadro 1) e 203 não constantes ( $\mathrm{IC} \geq 0,80$ ) (Quadro 2).

\section{Discussão}

A terminologia na Enfermagem tem relevante contribuição para a profissão, visto que seu devido uso e reconhecimento contribuem para o aumento da capacidade de raciocínio clínico tão importante na prática cotidiana assistencial da classe, já que proporciona vantagens para um registro qualificável e direcionado, possibilitando o desenvolvimento de um vocabulário específico da área e, dessa forma, na busca por redução de ambiguidade e no incentivo para a completude da sistemática do cuidado. ${ }^{(13)}$

Os registros de Enfermagem não devem ser vistos apenas como uma parte burocrática do trabalho do enfermeiro, sendo necessária uma conscientização profissional da sua importância e das consequências 
Quadro 1. Termos constantes na CIPE ${ }^{\circledR}$ versão 2019

\begin{tabular}{|c|c|}
\hline Eixos CIPE ${ }^{\circledR}$ & Termos constantes na CIPE ${ }^{\circledR}$ versão 2019 \\
\hline Foco & 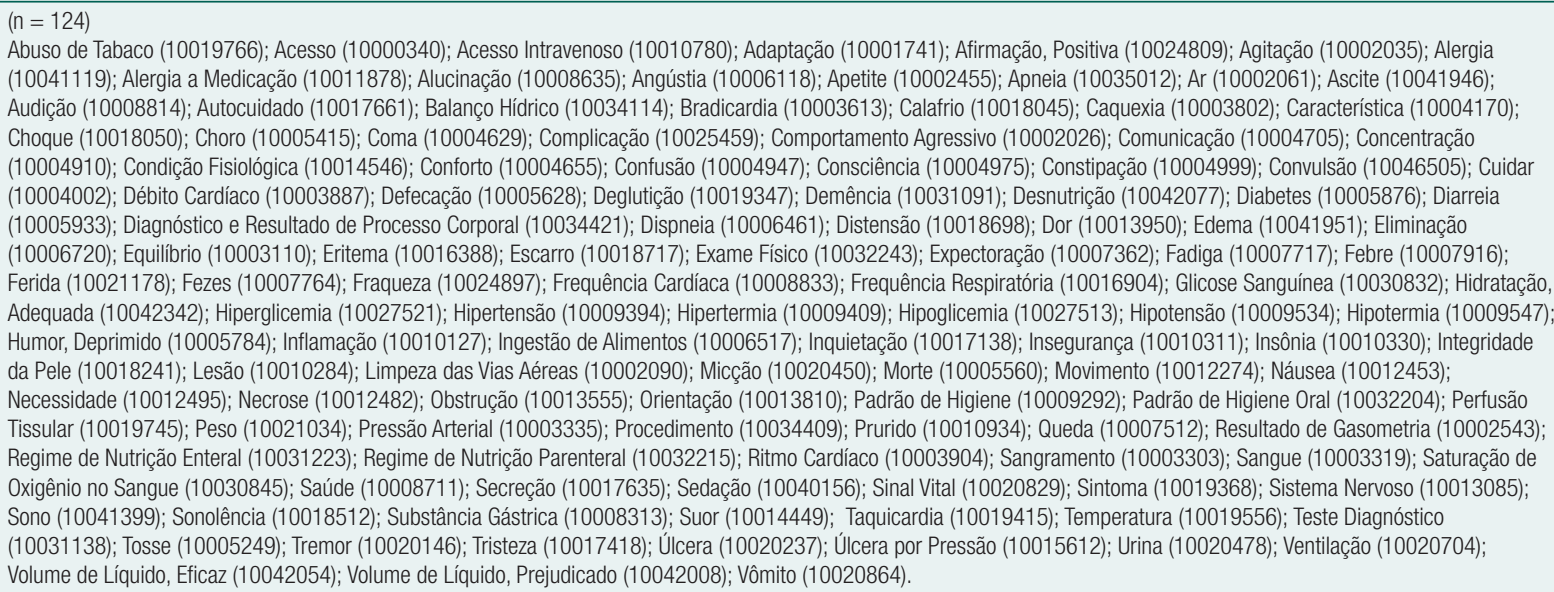 \\
\hline Julgamento & $\begin{array}{l}(n=18) \\
\text { Anormal (10013269); Dependência (10026671); Estado (10018786); Extensão (10007423); Grande (10011116); Independência (10026721); Iniciado (10018764); Leve } \\
\text { (10025854); Melhorado (10026692); Moderado (10025865); Normal (10013295); Parcial (10014081); Pequeno (10018315); Prejudicado (10012938); Prescrito (10015506); } \\
\text { Presença (10046624); Progresso (10015789); Real (10000420). }\end{array}$ \\
\hline Meios & $\begin{array}{l}(n=55) \\
\text { Acesso Venoso Central (10004115); Alimento (10008089); Analgésico (10002279); Antibiótico (10002383); Antipirético (10037253); Aparelho de Sucção (10019029); Bandagem } \\
\text { (10003123); Cadeira de Rodas (10021052); Cama (10003168); Cateter (10004087); Cateter Urinário (10020373); Chuveiro (10018084); Cirurgia (10019212); Cirurgião } \\
\text { (10019190); Coletor de Urina (10020484); Curativo (10021227); Compressas/Gazes (10008378); Contensão (10017164); Creme (10005352); Dispositivo (10005869); } \\
\text { Dispositivo para Cobertura (10005306); Dispositivo Respiratório (10016958); Droga (10006314); Lençol Móvel (10006248); Enema (10006881); Enfermeiro (10013333); Equipo } \\
\text { para Soro (10006295); Fisioterapeuta (10024003); Fisioterapia (10036434); Fralda (10005914); Hemodiálise (10008949); Insulina (10010400); Luvas (10008487); Marca-passo } \\
\text { (10013945); Máscara (10011752); Máscara de Oxigênio (10013909); Medicação (10011866); Médico (10014522); Terapia Inalatória (10010213); Oxigenoterapia (10013921); } \\
\text { Oxímetro de Pulso (10032551); Plano (1001463); Prontuário do Paciente (10014178); Protocolo (10015926); Roupa de Cama (10003175); Serviço Social (10018475); Solução } \\
\text { (10018499); Sutura (10019323); Técnica (10019525); Técnica Asséptica (10002639); Técnica de Deambulação (10002222); Terapia Intravenosa (10010808); Tubo Endotraqueal } \\
\text { (10006868); Tubo Gastrointestinal (10008350); Vaso Sanitário (10004693). }\end{array}$ \\
\hline Ação & $\begin{array}{l}\text { (n=50) } \\
\text { Administrar (10001773); Agendar (10017528); Aliviar (10002171); Alterar (10002185); Aplicar (10002464); Aspirar (10002641); Aumentar (10009961); Auscultar } \\
\text { (10003012); Autorizar (10003020); Auxiliar (10002850); Colaborar (10004542); Coletar (10004574); Confirmar (10020727); Consultar (10005017); Contatar (10005038); } \\
\text { Controlar (10005142); Desenvolver (10005848); Diminuir (10005600); Drenar (10006211); Elevar (10006691); Encaminhar (10016576); Entubar (10010831); Estabilizar } \\
\text { (10018729); Estimular (10018842); Extrair (10006230); Higienizar (10009285); Informar (10010162); Inserir (10010324); Instalar (10010353); Limpar (10004444); Manter } \\
\text { (10011504); Massagear (10011768); Medir (10011813); Mobilizar (10012120); Notificar (10001917); Observar (10013474); Obter (10013572); Permitir (10014408); } \\
\text { Preparar (10015478); Prescrever (10015510); Promover (10015801); Prover (10015935); Puncionar (10016152); Regular (10016613); Requerer (10016873); Traçar } \\
\text { (10019967); Transferir (10020030); Tratar (10020133); Trocar (10004162); Verificar (10011813). }\end{array}$ \\
\hline Tempo & $\begin{array}{l}(n=18) \\
\text { Admissão (10001843); Agudo (10001739); Alta (10006000); Amanhã (10019811); Contínuo (10005086); Crônico (10004395); Dia (10005502); Duração (10006379); Encontro } \\
\text { (10006810); Exame (10007241); Presente (10015581); Semana (10021010); Frequência (10008234); Início (10013689); Manhã (10012226); Noite (10013207); Tarde } \\
\text { (10001955); Visita (10020817). }\end{array}$ \\
\hline Localização & 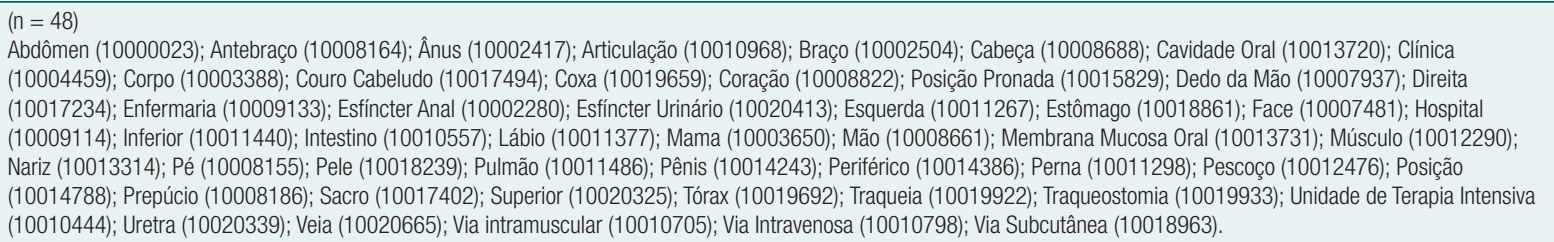 \\
\hline Cliente & $\begin{array}{l}(n=6) \\
\text { Adulto (10001889); Família (10007554); Idoso (10006604); Irmã (10021653); Mãe (10027257); Paciente (10014132). }\end{array}$ \\
\hline
\end{tabular}

do não preenchimento correto das documentaçóes existentes. Por outro lado, entre as limitaçóes relacionadas ao efetivo registro, encontram-se a falta de recursos humanos, falta de tempo hábil para realizar a documentação, falta de interesse institucional e a cultura de que a enfermagem é um serviço de suporte aos outros profissionais de saúde. ${ }^{(20)}$

Todavia, neste estudo, os resultados apontaram uma boa qualidade de registros e, após ma- peamento, concluiu-se que 319 termos estavam constantes na CIPE versão 2019, o que denota que os enfermeiros podem estar acompanhando a literatura e aplicando termos em sua prática diária, reforçando, assim, sua escrita com base em evidências científicas. Estudo ${ }^{(11)}$ que identificou termos para mulheres idosas com HIV/aids a partir de um documento especializado do Ministério da Saúde evidenciou uma abrangência maior também nos 
Quadro 2. Termos não constantes na CIPE ${ }^{\circledR}$ versão 2019

\begin{tabular}{|c|c|}
\hline Eixos CIPE ${ }^{\circledR}$ & Termos não constantes na CIPE ${ }^{\oplus}$ versão 2019 validados \\
\hline Foco & $\begin{array}{l}\text { (n = 58) } \\
\text { Abscesso; Anasarca; Anorexia; Anúria; Apatia; Assistolia; Astenia; Bem-estar; Broncoaspiração; Bulha Cardíaca; Cefaleia; Cianose; Cicatriz; Coágulo; Colesterol; Coma Induzido; } \\
\text { Comorbidade; Corrimento Vaginal; Crepitaçôes; Débito Urinário; Deiscência; Dermatite; Dieta; Dieta líquida; Eupneia; Exsudato; Flebite; Fluído; Fraçãoo Inspiratória de Oxigênio; } \\
\text { Hematúria; Hemiparesia; Hemotórax; Hipocromia; Hipotonia; Icterícia; Impaciência; Ingestão; Isocoria; Jejum; Líquido; Lucidez; Melena; Murmúrio vesicular; Normocardia; } \\
\text { Normotermia; Nutrição Enteral; Palidez; Pneumatose Intestinal; Pressão Expiratória Final Positiva; Pulso; Repouso; Restrição; Ruídos Adventícios; Sopro Cardíaco; Taquipneia; Tecido } \\
\text { de Granulação; Tecido Fibroso; Torpor; Tosse Produtiva. }\end{array}$ \\
\hline Julgamento & $\begin{array}{l}(n=11) \\
\text { Ausente; Deficiência; Deficiência Motora; Deficiente; Distendido; Estável; Flácido; Globoso; Grave; Preservado; Satisfatório. }\end{array}$ \\
\hline Meios & $\begin{array}{l}\text { (n=78) } \\
\text { Ácido Graxo Essencial; Água Estéril para Injeção; Alginato de Cálcio; Ampola; Ansiolitico; Antiarrítmico; Antibiograma; Antidiarreico; Antiemético; Antiespasmódico; Antiflatulento; } \\
\text { Antifúngico; Anti-hipertensivo; Anti-infeccioso; Antiretroviral; Banho; Banho de Aspersão; Banho no Leito; Benzoato de Benzila; Benzodiazepínico; Bicarbonato; Bomba de Infusão; } \\
\text { Cabeceira; Cadeira; Cadeira de Banho; Cardiologista; Cateter Venoso; Cloreto de Potássio; Clorexidina Degermante; Colagenase; Colchonete Estático de Ar; Comprimido; } \\
\text { Concentrado de Hemácias; Concentrado de Plaquetas; Contraste lodado; Corticoide; Cultura de Bacilo de Koch; Curativo Oclusivo; Curativo Úmido; Dispositivo Urinário; Droga } \\
\text { Vasoativa; Eletrocardiograma; Escala de Glasgow; Escala de Ramsay; Esfigmomanômetro; Esparadrapo; Exame de Sangue; Extensor Intermediário; Frasco; Funcionário; } \\
\text { Gastrostomia; Hemocomponente; Hemocultura; Hemoderivado; Hidrocolóide; Hidrogel; Infusão; Manta Térmica; Maqueiro; Papaína; Parasitológico de Fezes; Plantão; Plasma; } \\
\text { Pleurotomia; Pomada; Prescriçãa Médica; Raio X; Solução Alcoólica; Solução Fisiológica; Solução Glicosada; Técnico de Enfermagem; Terapia de Reidratação Oral; Tomografia } \\
\text { Computadorizada; Transfusão Sanguínea; Tricotomia; Ultrassonografia; Urocultura; Ventilação Mecânica. }\end{array}$ \\
\hline Ação & $\begin{array}{l}(n=23) \\
\text { Abrir; Aceitar; Aguardar; Antecipar; Apresentar; Chamar; Consumir; Cooperar; Desligar; Fechar; Fixar; Indicar; Introduzir; Liberar; Modificar; Mudar; Permanecer; Preencher; } \\
\text { Procurar; Realizar; Suspender; Utilizar; Verbalizar. }\end{array}$ \\
\hline Tempo & $\begin{array}{l}(\mathrm{n}=5) \\
\text { Diário; Frequente; Horário; Momento; Súbito. }\end{array}$ \\
\hline Localização & $\begin{array}{l}\text { (n=23) } \\
\text { Ambiente; Ambulatório; Banheiro; Cavidade; Coluna Vertebral; Decúbito Lateral; Farmácia; Garganta; Glúteo; Jejunostomia; Laboratório; Linfonodo; Meato Urinário; Membro Inferior; } \\
\text { Membro Superior; Mucosa; Necrotério; Posição de Fowler; Pronto-socorro; Pupila; Região inguinal; Veia jugular; Via Aérea Superior. }\end{array}$ \\
\hline Cliente & $\begin{array}{l}(\mathrm{n}=5) \\
\text { Acompanhante; Cliente; Esposa; Filho; Jovem. }\end{array}$ \\
\hline
\end{tabular}

termos constantes na $\mathrm{CIPE}^{\circ}$, corroborando com o presente estudo.

Cumpre salientar que a pesquisa para mulheres idosas com HIV/aids ${ }^{(11)}$ trouxe similaridades ao estudo atual já que 20 termos foram encontrados em ambas as pesquisas, quais sejam: "Autocuidado", "Cuidar", "Diagnóstico e resultado", "Lesão", "Morte", "Necessidade", "Saúde" (eixo foco), "Presença" (eixo Julgamento), "Droga" (eixo Meios), "Aplicar", "Colaborar", "Consultar", "Controlar", "Desenvolver", "Encaminhar", "Notificar", "Promover", "Tratar" (eixo Açáo), "Corpo" (eixo Localização) e "Família", "Idoso" (eixo Cliente). Apesar do primeiro estudo ter partido de um publicação do Ministério da Saúde e o segundo dos registros de enfermeiros, os termos comuns refletem aspectos biológicos do cuidado às pessoas vivendo com aids.

Dentre os eixos existentes, o eixo "Foco" abrangeu a maior frequência de termos constantes na $\mathrm{CIPE}^{\circ}$, no presente estudo, justificado pelo fato deste apresentar as principais áreas de atençáo relevante para a enfermagem. Este fato demonstra que os enfermeiros pontuam situaçóes importantes em seus registros e que dignificam em âmbito prático.

No eixo "Foco", o termo constante que apresentou uma frequência considerável foi "Sinal Vital", já entre os não constantes destacou-se "Dieta". Os sinais vitais são considerados indicadores clínicos das condiçôes hemodinâmicas de qualquer paciente, sendo a pressão arterial sistêmica, a frequência respiratória, o pulso/frequência cardíaca, temperatura e dor, os mais verificados na prática clínica. ${ }^{(28)}$

As pessoas vivendo com aids apresentam flutuaçôes nos seus padróes clínicos, uma vez que a doença promove a imunossupressão das defesas, deixando o paciente suscetível às diversas enfermidades. Como exemplo, os episódios de hipertermia que possam estar vinculados ao aumento da viremia, exacerbando a produção de substâncias que afetam o sistema termorregulador. E assim tal quadro pode afetar outros sistemas, como o cardiovascular, mudando a sua dinâmica fisiológica. ${ }^{(29)}$

A dieta adequada às pessoas vivendo com aids no ambiente hospitalar é imprescindível para a redução das repercussōes do estresse fisiológico, a prevenção ou o tratamento da desnutriçăo, a recuperação do indivíduo a longo prazo e a melhora da qualidade de vida. É factível pontuar que esses pacientes têm perdas nutricionais significativas que provêm dos mecanismos patológicos, por exemplo, o aumento do catabolismo que possa estar relacionado com a carga viral do indivíduo. Ademais, o uso dos medi- 
camentos antirretrovirais que liberam coenzimas e assim mudam o $\mathrm{pH}$ do suco das papilas gustativas afetam o paladar dos pacientes. Sendo assim, a enfermagem tem papel fundamental no sucesso dessa terapêutica, uma vez que é ela a responsável pela administração da dieta; acesso do trato gastrointestinal, quando prescrito; manutenção desta via, e resposta frente às intercorrências. ${ }^{(30)}$

No eixo "Julgamento", observou-se em maior frequência como constante na $\mathrm{CIPE}^{\circ}$ o termo "dependência", indicando o estado de comprometimento das pessoas vivendo com aids assistidas no ambiente hospitalar. Quanto mais tardio for o diagnóstico, maior serão as complicaçóes relacionadas à doença e a necessidade de ajuda para o desempenho de atividades básicas da vida diária, como alimentação, banho, vestir-se e despir-se e administração de medicamentos. ${ }^{(29)}$

Já o termo "estável", não constante na CIPE", reflete a condiçáo do paciente e denota a impotência da avaliaçáo do seu estado de saúde durante o seu período de internação. A identificação correta dos problemas apresentados pelos pacientes, por meio de uma avaliação clínica cuidadosa, torna-se fundamental para o desenvolvimento das açóes do enfermeiro. ${ }^{(5,6)}$

No eixo "Meios", destacaram-se os termos "Medicação", constante, e "Banho de Aspersão", não constante na CIPE:. A medicação é de suma importância para a terapêutica das pessoas vivendo com aids, uma vez que necessitam fazer uso da terapia antirretroviral (TARV). A não adesão ao uso da TARV pode ser considerada uma ameaça nos planos individual e coletivo, levando ao comprometimento da efetividade da terapia medicamentosa e favorecendo a disseminação de vírus que apresentem resistência aos medicamentos disponíveis, respectivamente, além do impacto para as políticas públicas de oferta de medicamentos antirretrovirais e para o sistema de saúde. ${ }^{(29)}$ Assim sendo, destacam-se o papel do enfermeiro como primordial na supervisão do uso da medicação, aconselhamento ao paciente e intervençóes adaptadas à realidade de cada usuário, contribuindo para redução da mortalidade e melhoria da qualidade de vida dessas pessoas. ${ }^{(5,6)}$
O banho de aspersão é recomendado enquanto medida de estímulo ao autocuidado. Isso se deve ao seu potencial de alcançar ganhos funcionais, na promoção de autogestão independente e desempenho contínuo das atividades de vida diária. ${ }^{(31)}$

No eixo "Ação" o termo constante na $\mathrm{CIPE}^{\oplus}$ mais frequente foi "Administrar", já entre os não constantes destacou-se "Aceitar". O termo administrar está relacionado, principalmente, à TARV, dieta e cuidados de enfermagem às pessoas com aids hospitalizadas. No tocante às características da dieta para esses pacientes, recomenda-se a hipercalórica e hiperproteica, a qual contribui na diminuição da desnutrição e melhora do quadro clínico. ${ }^{(29)}$

$\mathrm{O}$ esquema antirretroviral inicial preconizado pelo Ministério da Saúde é composto pelas drogas tenofovir, lamivudina e dolutegravir. Este tem como objetivo impedir a replicação viral, reduzindo, consequentemente, a carga viral e diminuindo, assim, a mortalidade dos pacientes. ${ }^{(29)} \mathrm{O}$ enfermeiro deve possuir domínio acerca do esquema, dose, frequência e efeitos adversos, priorizando, dessa forma, a segurança do paciente, o que é um princípio fundamental do cuidado.

Já o termo "Aceitar" relaciona-se à medicação prescrita, dieta e ao diagnóstico da doença. Nesse contexto, o enfermeiro pode contribuir na promoção da aceitação desse diagnóstico por parte do paciente, orientaçôes necessárias diante das modificações impostas pela nova condição de saúde, no tratamento e ações de educação em saúde. ${ }^{(3)}$ Ademais, a "aceitação" é uma estratégia de enfrentamento/ adaptação psicológica que contribui para a superação e autocuidado das pessoas vivendo com aids. ${ }^{(32)}$

Em relação ao eixo "Tempo", identificaram-se os termos "Admissão" e "Horário" como mais frequentes. Por sua vez, a "Admissão", termo constante na CIPE", representa um momento importante para coletar dados, facilitar a adaptação do paciente ao ambiente hospitalar, além de proporcionar conforto e segurança. Ademais, informaçôes obtidas na admissão possibilitam o acompanhamento do cliente até o momento da sua alta.

O termo "Horário" está ligado aos cuidados de enfermagem e seus procedimentos técnicos, padronizados de acordo com a instituição. As ações 
encontradas na prescrição precisam ser verificadas após a execução do cronograma de prestação de cuidado, como verificação de sinais vitais, administração de medicações, mudança de decúbito e tipo de dieta, permitindo, assim, um cuidado integral dos pacientes com aids. ${ }^{(3)}$

No eixo "Localização", houve o predomínio do termo "Direita", constante na CIPE", e do termo "Banheiro", não constante nesse sistema de classificação. O termo "Direita" nos registros de enfermagem refere-se à estrutura corporal e estava estritamente vinculado ao local das punçóes venosas, tanto central quanto periférico, que devido ao protocolo específico da unidade onde os dados foram coletados, são prioritariamente realizadas em lado direito e membro superior, respectivamente. Estudo prospectivo realizado em unidade hospitalar no Sudeste do Brasil identificou como principal local de punção, a veia basílica direita, seguida da braquial direita. ${ }^{(33)}$

O termo "Banheiro" correspondente ao local de realizaçáo de higiene do paciente, sendo uma das prescriçóes de cuidado do Enfermeiro na instituição, para os que se encontram em condiçóes de realizá-lo, no intuito de estimular o autocuidado e sua independência.

No que diz respeito ao eixo "Cliente", a utilização do termo constante, "Paciente", bem como do não constante, "Cliente", demonstra que a assistência prestada era focada, sobretudo, nesse indivíduo, não sendo mencionada a participação da família no cuidado, nos registros de enfermagem analisados no referido hospital. Ademais, o termo paciente é um vocábulo muito frequente dentro do ambiente hospitalar, sugerindo uma posição que indica passividade em relação ao profissional de saúde.

É importante destacar como potencialidade, que o estudo atual validou 203 termos não constantes na $\mathrm{CIPE}^{\oplus}$, enquanto pesquisa realizada sobre banco de termos para a prática de enfermagem com mulheres idosas com HIV/aids, ${ }^{(11)}$ apenas 69 termos não constantes foram validados, e isso pode estar relacionado à magnitude metodológica dada a esta investigação, já que esta se valeu de análise de registros de enfermeiros de 65 prontuários.

Estudo que buscou definir termos não constantes na $\mathrm{CIPE}^{\circ} 2015$ para a prática de Enfermagem com mulheres idosas com vulnerabilidades ao HIV/ aids refere que, mesmo não estando inseridos na $\mathrm{CIPE}^{\circ}$, os termos traduzem fatores determinantes da clientela que foram identificados na temática de interesse voltada a uma população que necessita de assistência de Enfermagem eficaz e emancipatória. Sendo assim, esses termos caracterizam, com maior precisão, as especificidades dessa população, representando uma contribuição para a estruturação verbal de diagnósticos, resultados e intervençóes de Enfermagem. ${ }^{(34)}$

Portanto, o alto número de termos não constantes validados neste estudo indica um avanço na terminologia de enfermagem no cuidado à pessoa que vive com HIV/aids já que progrediu com termos decorrentes de uma vivência que é única. $\mathrm{O}$ fato das versôes atualizadas da $\mathrm{CIPE}^{\circ}$ contemplarem novos termos demonstra a relevância da constante atualização de uma classificação que tem por objetivo representar, universalmente, a prática de enfermagem. Ressalta-se que sistemas padronizados de registros de elementos da prática de enfermagem baseados na CIPE exigem adequação às revisóes da classificação, de modo a não se tornarem obsoletos. ${ }^{(34)}$

Diante dessas perspectivas, os termos serão base para a construção de enunciados de diagnósticos, resultados e intervençóes de enfermagem, caracterizando, assim, um progresso na nomenclatura da enfermagem, bem como subsidiando o avanço da profissão, pois fará parte de um incentivo para fases seguintes da construção de um subconjunto terminológico que atenda o ser humano em toda sua forma integral. ${ }^{(35)}$

Por conseguinte, os termos aqui apresentados poderão contribuir para uma abordagem sistemática de cuidados de enfermagem na prática clínica, dando maior clareza e facilidade em sua implementação, quando transformados em enunciados para o processo de enfermagem. $\mathrm{E}$ isso é possível pela utilização de dados empíricos observáveis e pela determinação de conceitos, dando direcionamento e favorecendo a tomada de decisão do profissional de enfermagem sobre suas açóes. ${ }^{(35)}$

A limitação da pesquisa se refere a não utilização de softwares para a extraçáo dos termos. No entanto, realizou-se um treinamento prévio dos pesquisadores buscando-se manter um rigor metodológico. 


\section{Conclusão}

A pesquisa permitiu a construção de uma terminologia especializada de enfermagem para o cuidado de pessoas adultas com aids, prevalecendo os termos constantes na CIPE ${ }^{\circ}$ 2019, nos eixos Foco e Meios. Ressalta-se que também identificou-se um número significativo de não constantes, para os quais, será sugerida a inclusão ao Conselho Internacional de Enfermeiros em versão futura dessa Classificação. Como potenciais estudos para pesquisas futuras, possibilitará a estruturação de um subconjunto terminológico da $\mathrm{CIPE}^{\circ}$ direcionado ao cuidado das pessoas vivendo com aids, bem como instrumentos clínicos, identificação de indicadores de enfermagem e modelos conceituais para essa clientela. A extração dos termos dos registros de enfermeiros destaca-se como uma potencialidade desta pesquisa, uma vez que reflete particularidades do cuidado de enfermagem direcionado a essa população. Ademais, contribui para o avanço do conhecimento relacionado à utilização da $\mathrm{CIPE}^{\oplus}$ no cuidado de enfermagem às pessoas com aids, colaborando, assim, para uniformização da linguagem profissional e desenvolvimento da ciência Enfermagem, além de prover subsídios para ensino e educação permanente, com foco na segurança do paciente.

\section{Agradecimentos}

Pesquisa financiada pelo Conselho Nacional de Desenvolvimento Científico e Tecnológico (CNPq), por meio da Chamada Universal 01/2016 - Faixa A, processo no 402978/2016-1 - "Diagnósticos/resultados e intervençóes de enfermagem da $\mathrm{CIPE}^{\circledast}$ para pessoas vivendo com Aids: estruturação de um subconjunto terminológico".

\section{Colaborações}

Silva BCO, Santos RM, Santos FR, Padilha TMS, Moreira OAA, Tavares ES e Silva RAR declaram que contribuíram com a concepção do estudo, análise e interpretação dos dados, redação do artigo, revisão crítica relevante do conteúdo intelectual e aprovação da versão final a ser publicada.

\section{Referências}

1. Avong YK, Jatau B, Gurumnaan R, Danat N, Okuma J, Usman I, et al. Addressing the under-reporting of adverse drug reactions in public health programs controlling HIV/AIDS, tuberculosis and malaria: a prospective cohort study. PLoS One. 2018;13(8):e0200810.

2. Joint United Nations Program on HIV/AIDS Brasil. Estatísticas. Brasília: Joint United Nations Program on HIV/AIDS Brasil; 2019.

3. Souza Neto VL, Silva RA, Silva CC, Negreiros RV, Rocha CC, Nóbrega MM. [Proposal of nursing care plan in people hospitalized with AIDS]. Rev Esc Enferm USP. 2017:51(e03204):1-9. Portuguese.

4. Rocha GS, Angelim RC, Andrade AR, Aquino JM, Abrão FM, Costa AM. [Nursing care of HIV-positive patients: considerations in the light of phenomenology]. Rev Min Enferm. 2015;19(2):258-61. Portuguese.

5. Conselho Federal de Enfermagem (COFEN). Resolução $n^{0} 358$ do Conselho Federal de Enfermagem, de 15 de outubro de 2009. Brasília (DF): COFEN; 2009.

6. Leal RG, Bastos CR, Rodrigues AL, Pires SM, Carvalho DR, Cubas MR. Validation of the definition of terms identified in electronic records of nursing of a university hospital]. Cienc Cuid Saude. 2017;16(1):1-8. Portuguese.

7. Garcia TR. [ICNPR: a standardized terminology to describe professional nursing practice] Rev Esc Enferm USP. 2016;50(3):376-79. Portuguese.

8. Strudwick G, Hardiker NR. Understanding the use of standardized nursing terminology and classification systems in published research: A case study using the International Classification for Nursing Practice ${ }^{(囚)}$. Int J Med Inform. 2016;94:215-21.

9. Bittencourt GK, Siqueira MC, Beserra PJ, Nóbrega MM, Nogueira JA, Silva AO. [Mapping of nursing diagnosis for elderly women in the context of vulnerability to HIV/AIDS]. Rev Enferm UFPE on line. 2015;9(4):7364-74. Portuguese.

10. Tannure MC. Sistematização da Assistência de Enfermagem - Guia prático. 2a ed. Rio de Janeiro: Guanabara Koogan; 2010.

11. Siqueira MC, Bittencourt GK, Nóbrega MM, Nogueira JA, Silva AO. [Term base for nursing practices with elderly women with HIV/AIDS]. Rev Gaúcha Enferm. 2015;36(1):28-34. Portuguese.

12. Carvalho CM, Cubas MR, Nóbrega MM. Terms of the specialized nursing language for the care of ostomates]. Rev Bras Enferm. 2017;70(3):461-7. Portuguese.

13. Duarte FH, Santos WN, Silva FS, Lima DM, Fernandes SF, Silva RA. [Terms of specialized nursing language for people with pressure injury]. Rev Bras Enferm. 2019;72(4):1028-35. Portuguese.

14. Oliveira MD, Lima J0, Garcia TR, Bachion MM. [Useful terms for nursing practice in the care of people with leprosy]. Rev Bras Enferm. 2019;72(3):744-52. Portuguese.

15. Andrade LL, Costa KN Nóbrega MM, Oliveira JS, Accioly CM. [Termos identificados em uma clínica médica e classificados como não constantes na CIPE ${ }^{\circledR}$. Rev Eletr Enf. 2012;14(2):330-6.

16. Norat EM, Trigueiro EV, Nóbrega MM, Garcia TR. Banco de termos da linguagem especial de enfermagem da clínica cirúrgica de um hospital universitário. J Nurs UFPE online. 2009;3(2):204-14. 
17. Nóbrega MM, Garcia TR, Nóbrega RV, Araújo RT. Banco de termos da linguagem especial de enfermagem da unidade de terapia intensiva neonatal de um hospital de ensino. Online Braz J Nurs. 2009;8(2):116. Portuguese.

18. Souza DR, Andrade LT, Napoleão AA, Garcia TR, Chianca TC. [Terms of International Classification for Nursing Practice in motor and physical rehabilitation]. Rev Esc Enferm USP. 2015;49(2):2009-15. Portuguese.

19. Silva RS, Nóbrega MM, Medeiros AC, Jesus NV, Pereira A. [Terms of the ICNP® used by the team of nurses assisting people in palliative cares]. Rev Eletr Enf. 2015;17(2):269-77. Portuguese.

20. Gomes DC, Cubas MR, Pleis LE, Shmeil MA, Peluci AP. Terms used by nurses in the documentation of patient progress]. Rev Gaúcha Enferm. 2016;37(1):e53927:1-8. Portuguese.

21. Fontelles MJ, Simões MG, Almeida JC, Fontelles RG. Metodologia da pesquisa: diretrizes para o cálculo do tamanho da amostra. Rev Para Med. 2010;24(1):57-64.

22. Lopes MV, Silva VM, Araujo TL. Validação de diagnósticos de enfermagem: desafios e alternativas. Rev Bras Enferm. 2013;66(5):649-55.

23. International Organization for Standardization (ISO). ISO 12300: health informatics: principles of mapping between terminological systems. Genebra: ISO; 2016.

24. Tannure MC, Chianca TC, Garcia TR. Construção de um banco de termos da linguagem especial de enfermagem. Rev Eletr Enf. 2009;11(4):1026-30.

25. Lenz E. Basic principles of measurement: operationalizing nursing concepts. In: Waltz CF, Strickland OL, Lenz E. Measurement in nursing and health research. 5th ed. New York: Springer Publishing Company; 2017.

26. Horta WA. Processo de enfermagem. Rio de Janeiro: Guanabara Koogan; 2011.
27. Batista CG, Matos MM. 0 acordo entre observadores em situação de registro cursivo: definições e medidas. Psicologia. 1984;10(3):57-69.

28. Teixeira CC, Boaventura RP, Souza AC, Paranaguá TT, Bezerra AL, Bachion $\mathrm{MM}$, et al. Vital signs measurement: an indicator of safe care delivered to elderly patients]. Texto Contexto Enferm. 2015;24(4):1071-8. Portuguese.

29. Brasil. Ministério da Saúde. Secretaria de Vigilância em Saúde. Departamento de Vigilância, Prevenção e Controle das Infecções Sexualmente Transmissíveis, do HIV/aids e das Hepatites Virais. Protocolo clínico e diretrizes terapêuticas para manejo da infecção pelo HIV em adultos. Brasília (DF): Ministério da Saúde; 2018.

30. Miranda RN, Kauffmann LKO, Pinto AF, Gonçalves RSL, Guterres AS. Caracterização antropométrica, dietética e alterações gastrointestinais de pacientes hospitalizados imunodeprimidos. Para Res Med J. 2019;3(2):e24:1-9.

31. Prado AR, Ramos RL, Ribeiro OM, Figueiredo NM, Martins MM, Machado WC. [Bath for dependent patients: theorizing aspects of nursing care in rehabilitation]. Rev Bras Enferm. 2017;70(6):1408-13. Portuguese.

32. Silva RT, Silva RA, Rodrigues ID, Souza Neto VL, Silva BC, Souza FM. [Coping strategies of people living with aids in face of the disease] Rev Latino-Am. Enfermagem. 2018;26(e2985)1-8. Portuguese.

33. Santo MKD, Takemoto D, Nascimento RG, Nascimento AM, Siqueira $\mathrm{E}$, Duarte CT, et al. Peripherally inserted central venous catheters: alternative or first choice vascular access?. J Vasc Bras. 2017 ;16(2):104-12.

34. Ferreira KS, Santos MC, Beserra PJ, Bittencourt GK. [Definition of nonconstant terms in the international classification for nursing practice for elderly women with vulnerabilities to HIV/AIDS]. Rev Enferm UFPE on line. 2017;11(11):4424-34. Portuguese.

35. Silva CL, Cubas MR, Silva LL, Cabral LP, Grden CR, Nichiata LY. Nursing diagnoses associated with human needs in coping with HIV. Acta Paul Enferm. 2019;32(1):18-26. 